\title{
Adults thinking the way we think children think, but children don't always think that way: A study of perceptual salience and problem solving
}

\author{
RICHARD D. ODOM, JOSEPH G. CUNNINGHAM, and EILEEN C. ASTOR \\ Vanderbilt University, Nashville, Tennessee 37240
}

\begin{abstract}
Adults and third-grade children were given a series of tasks requiring the use of cognitive operations associated with traditional conservation problems. While the adults showed an inability to solve the critical problem in the series, the children solved it easily except in the case when the irrelevant information was made highly salient. These results were interpreted as supporting an account emphasizing perceptual change rather than traditional cognitive change accounts of the development of problem solving.
\end{abstract}

In tasks used in developmental research on human problem solving, the subject is provided with several alternative choices, consisting of one that is relevant to obtaining the goal and one or more that are goal irrelevant. The goal-relevant alternative is always predetermined by an adult experimenter who considers its increasingly reliable choice to be a reflection of problem solving.

Among the published reports in this broad field of research, it is unusual to find younger children performing at an equivalent or higher level than older children or adults. Rather, increasing developmental level is typically accompanied by more accurate and more rapid problem solving. Such results are usually explained in terms of qualitative or quantitative changes in cognition, and the extant accounts may be generally characterized as emphasizing changes in processes that analyze, evaluate, and/or transform stored and externally based information. Examples of commonly proposed constructs that have been used to account for developmental change are (a) verbal mediators (Kendler \& Kendler, 1962), (b) attentional processes (Hagen \& Hale, 1973), and (c) structures of logical thought (Piaget \& Inhelder, 1969).

In these cognitive-change positions there is an implicit assumption that all information in a problem-solving task is perceived in the same way by subjects at all developmental levels. Consequently, age-related performance differences are considered to result from different cognitive structures that analyze and evaluate the same information.

The authors wish to thank Frank B. Murray for introducing them to the bead problem. To Jennie Fox and Carlton Odom, two young children who served as pilot subjects and convinced us with their competence that this study would be worth doing, we owe a special debt of gratitude. We are also grateful to the children and staff of Ensworth School for their splendid help and cooperation.
An alternative view that considers the evaluation of different rather than the same task information and focuses on developmental changes in the perceptual characteristics of that information has been advanced by Odom and Guzman (1972). This position emphasizes the role and development of the perceptual system in determining the detection and organization of task information for further processing through cognitive structures such as those described in cognitive-change positions.

Gibson (1969) maintains that relations and dimensions are present in the external environment; that they serve as basic information for the perceptual system and are not products of cognitive structures involving associations, images, or inferences; and that they are discovered in increasing numbers by the perceptual system as development proceeds. By extending these ideas, Odom and Guzman (1972) have proposed that the perceptual system becomes differentially sensitive to already discovered relations primarily as a function of varying perceptual experience per se and/or special environmental contexts and events. Therefore, as perceptual experience increases with development, perceptual sensitivity to most relations and categories would be expected to increase. Furthermore, it is proposed that the degree of sensitivity to relations or categories determines the order in which they are processed for problem solution through cognitive mechanisms that analyze, evaluate, and transform information. That is, the greater the perceptual sensitivity to given information, the higher the probability of its being cognitively processed, regardless of its appropriateness for problem solution. Therefore, with increasing development, an increasing amount of both relevant and irrelevant information would be cognitively processed and either accepted or rejected for problem solution.

This position has found support in research in which the relative sensitivity of the perceptual system to 
common relations and dimensions was assessed using traditional salience tasks prior to the presentation of problem-solving tasks in which the task information was comprised of such relations and dimensions (e.g., Odom, 1972; Odom, Astor, \& Cunningham, 1975; Odom \& Corbin, 1973). In a variety of tasks, it has been shown that when young children are given problems in which the goal-relevant information is high in salience, their performance reflects a level of cognitive competence that is often attributed only to older children.

Apart from salience research, however, there are few studies that demonstrate comparable competence across development in solving problems that are designed to assess cognitive structure. Even in those rare cases where younger subjects are shown to solve problems more efficiently than older subjects, the results are given traditional cognitive-change interpretations (e.g., Weir, 1964).

Preferences for cognitive change interpretations of psychological development may be an important cause for the dearth of research demonstrating comparability in cognitive structure across development. First, it is important to recognize that problems presented to children in research are often designed by adults who have such preferences. Second, as designer of the problem, it is unlikely that the adult would create one that he or she would find difficult to solve because of the expectation that someone younger would be likely to fail it. Identifying problems that younger subjects solve as well as or better than older subjects should be seriously considered as worthwhile. Such an approach could lead to a greater understanding of cognitive and perceptual development through considering not only the solution requirements of the task but also the perceptual characteristics of the task information.

In the present investigation, this approach was taken. Adults and children were verbally presented a problem that a number of adults, observed in informal settings, had failed to solve. The standard problem was as follows:

"Imagine that I have two cans. One has red beads in it, and it is called the red-bead can. The other has blue beads in it and is called the blue-bead can. There are the same number of red beads in the red-bead can as there are blue beads in the blue-bead can. Let me repeat that. There are the same number of red beads in the redbead can as there are blue beads in the blue-bead can. Now imagine that I dip a cup into the red-bead can and take out five beads. I pour them into the blue-bead can. Then I mix up all of the beads in the blue-bead can. I then dip the cup into the blue-bead can and take out five beads and pour them into the red-bead can. Will the number of red beads in the red-bead can and the number of blue beads in the blue-bead can be the same or different?"
This might be considered a type of conservation problem, and the correct answer to the question is "same." An acceptable explanation for this judgment would involve either adding and subtracting operations with specific numbers of colored beads or something general like "the number of red beads left in the bluebead can equals the number of blue beads taken to the red-bead can," which implies adding and subtracting operations. Solution to the task therefore requires that perceptually given information about the number and color of beads be evaluated through cognitive operations involving adding and subtracting.

In an attempt to understand why subjects would fail to give a "same" response to this problem, we surmised that the cognitive evaluation of information about the mixing of beads would result in probability estimates of, for example, returning fewer red beads to the redbead can than were originally taken from it because the sample would contain blue as well as red beads. However, whether mixing did or did not occur, a correct solution to the problem could be achieved by assuming that five beads of the same color were returned to the red-bead can. The mixing information, therefore, was irrelevant and unnecessary to solving the problem. Two studies were conducted to assess the role of this irrelevant information.

\section{STUDY 1}

In Study 1 each subject received three problems: the standard problem (mix), one in which the same beads were returned following no mixing (no mix), and one in which fewer beads were returned than had been originally taken (mix 1 less). The latter two problems were given to assess performance in similarly constructed tasks containing some information that was different and to assess the possibility of age-related response biases. All subjects were expected to give a "same" judgment to the no-mix problem and a judgment of "different" to the mix-1-less problem, since information about mixing was either absent or could be cognitively evaluated as irrelevant due to the additional information about the inequality of the number of beads taken and returned.

While the tasks in the salience research cited previously were primarily visual in nature, the tasks of the present study were presented verbally. It is reasonable, however, to assume that the perceptual system is differentially sensitive to the various content and structural relations that are contained in a linguistic context just as it is to relations in other contexts where salience has been shown to play a role.

In the standard mix problem, the salience of the mixing information was expected to be high for the adults and to be reflected in incorrect judgments resulting from their cognitive evaluation of that information. The mixing information was expected to be low 
in salience for the children because they had had no formal training in probability and sampling concepts and had had fewer years and opportunities than the adults to informally experience instances of them. Therefore, the children were expected to be less likely than the adults to make judgment errors as a result of cognitively evaluating the mixing information.

In the present study all subjects were probed for explanations or operations on which their judgments could have been based. In addition, confidence ratings of judgments were obtained for all subjects, and the younger subjects were probed for probability concepts.

\section{Method}

Subjects. The subjects were 20 Vanderbilt adults selected from an introductory psychology course and 20 third-grade children (mean $\mathrm{CA}=8$ yr 4 months) selected from a private school in Nashville.

Procedure. The three problems were read by an adult experimenter to each child individually and to groups of adults. The adults were given answer sheets on which to respond, whereas the children observed the experimenter record their responses on identical answer sheets. For half of the subjects in each developmental group the problems were presented in the order mix, no mix, mix 1 less, and for the remaining half in the order no mix, mix 1 less, and mix.

The no-mix and mix-1-less problems were identical to the standard mix problem except for variations introduced after the experimenter said, "I pour them into the blue-bead can." In the no-mix problem this was followed by, "I then dip the cup into the blue-bead can and take out the same five red beads and pour them into the red-bead can." In the mix-1-less problem the experimenter said, "Then I mix up all of the beads in the blue-bead can. I then dip the cup into the blue-bead can and take out only four beads and pour them into the red-bead can." The same question asked of the subject at the end of the mix problem was asked at the end of the other two problems. The order of the terms "same" and "different" in the question was counterbalanced across the problems.

Following a judgment to each problem, the subjects were asked to rate the confidence of their judgment. The adults indicated this on a 5-point scale, with categories varying from "very certain" (1) to "very uncertain" (5). The children were given a 3-point scale by the experimenter who asked, "Are you real sure (1), sort of sure (3), or not sure (5) about your answer?" The presentation order of these three categories was varied.

After judgments and confidence ratings had been made to each of the three problems, the experimenter read the mix problem once again. The adults were given $3 \mathrm{~min}$ to write down an explanation for their judgment. The children were asked to say why they had responded as they had and then were taken by the experimenter through the steps of the mix problem, which at this time was begun arbitrarily with six beads in each can. To assess subjects' competence in adding and subtracting operations, they were asked to state the number of each set of beads in each can after each step in the problem.

Following this, the experimenter probed the last 13 children for a concept of probability. This was done by posing a hypothetical situation in which many samples of five beads were dipped from the blue-bead can after the red beads had been mixed with the blue beads and asking whether there would be more cups with all red or all slue or whether there would be more with red and blue together.

\section{Results and Discussion}

The results were in accord with expectations. Only 3 of the 20 adults gave correct "same" judgments for the mix problem, while only 1 of the 20 children gave an incorrect judgment of "different." All of the subjects correctly answered both the no-mix and mix-1-less problems. The order of problem presentation had no effect on judgments. Both age groups expressed confidence in their judgments in that all mean confidence ratings were 2.0 or less, with 1 indicating highest certainty.

None of the three adults who gave "same" judgments for the mix problem gave a satisfactory explanation. Of the adults incorrectly responding with "different," only one corrected his judgment and gave a satisfactory explanation. In their explanations for judgments in the mix problem, 14 of the 20 adults gave probability accounts involving the mixing information.

The 19 children who gave a correct judgment also demonstrated adding and subtracting operations necessary for a satisfactory explanation when the experimenter took them through the steps of the mix problem. To the experimenter's initial question concerning why subjects had judged as they had, all but two children answered with something like "five beads were taken and five were returned." The one child who gave an incorrect judgment changed it and gave a satisfactory explanation. The mixing operation was clearly unimportant, and none of the children mentioned it in their explanations. Of the 13 children who were probed for a probability concept, $11(85 \%)$ indicated that there would be more cups containing both red and blue beads than cups with beads of a single color. Prior to the probe for a probability concept, 8 of the total sample of 20 children spontaneously indicated that the returning cup would have both red and blue beads in it. These data provide more than suggestive evidence that the mixing information was sufficiently salient to be perceived and that the effects of mixing were cognitively understood.

The children's performance therefore reflected cognitive operations and structures necessary for evaluating and analyzing information that was both relevant and irrelevant for problem solution. However, the irrelevant information was apparently so low in salience that it played no important role in determining their correct judgments. For the adults, on the other hand, it appeared to be quite salient and resulted in incorrect judgments.

\section{STUDY 2}

It might be argued that, even though the children in Study 1 showed evidence for those cognitive structures necessary for evaluating both the relevant and irrelevant information in the bead problem, they, unlike the adults, could have been deficient in coordinating operations. It might be reasoned that such a deficiency would prevent them from evaluating and coordinating both relevant and irrelevant information, even if the latter was also high in salience. 
This issue was investigated in the second study by giving children a mix problem that was modified to increase the salience of the irrelevant mixing information. This was accomplished by adding phrases related to mixing within the context of the standard problem.

If the subjects were deficient in coordinating operations, they would be expected either to perform like the children in Study 1 or to show no consistent pattern of judgments. If the salience manipulations effectively resulted in the coordination of the relevant and irrelevant information, then they would be expected to make judgments like those of the adults in Study 1 .

\section{Method}

The subjects were 10 third-grade children (mean $\mathrm{CA}=$ 8 yr 6 months) selected from the same school as the children in Study 1. The problem read to each child by the experimenter was identical to the mix problem in Study 1 except for the information presented after the experimenter said, "I pour them into the blue-bead can." Following this sentence the experimenter said, "Then I mix up all the beads in the bluebead can very well so that the red beads are mixed up with the blue beads. I then dip the cup into the blue-bead can and take out five beads; some of them are red and some of them are blue. I pour those five beads into the red-bead can." The experimenter recorded each child's answer to the standard question that came at the end of the problem and then obtained a confidence rating.

\section{Results and Discussion}

Only 1 of the 10 children solved the problem correctly, and the mean confidence rating was 2.6 . That is, $90 \%$ of the children in Study 2 gave a judgment of "different" just as $85 \%$ of the adults had done in the standard mix problem of Study 1. It appears, therefore, that the young child can be made to seem just as cognitively incompetent as the adult when irrelevant information is highly salient and likely to be evaluated for problem solution.

\section{CONCLUSIONS}

Age-related differences in the salience of given information are assumed to be due in large part to the number of past situations that provide experiences with that information. Because amount of exposure to information of all sorts is almost always positively correlated with age, more information would be expected to be highly salient for older subjects who would consequently be expected to evaluate (including accepting or rejecting) more of the available information (both solution relevant and irrelevant) in a problem than. younger subjects. Younger subjects may be relatively less accurate in most problem-solving tasks used in developmental research because they are cognitively evaluating highly salient, but irrelevant, information for problem solution. That is, their inaccuracy may not be primarily due to less developed cognitive structures and operations that analyze, evaluate, and transform information but to perceptual characteristics, like salience, of that information. Only in rare situations, such as the standard mix problem of Study 1, are younger subjects more accurate than older subjects. In such cases, however, it is doubtful that cognitivechange theorists, who give little or no attention to the role of perceptual development and to age-related differences in what information is likely to be processed cognitively, would be willing to conclude that, because of the older subjects' greater inaccuracy, they are less cognitively mature than the younger subjects.

The results of the present studies demonstrate how salience can reduce age-related performance differences that might otherwise be used as a basis for drawing cognitive-change conclusions. In conjunction with the results from other salience research, the present results serve to indicate the importance of the perceptual characteristics of information in problem-solving tasks. It is clear that, if we are to understand the development of the structure and function of cognitive processes that analyze, evaluate, and transform information, we must also understand the perceptual nature of that information and how it changes with development.

\section{REFERENCES}

Gibson, E. J. Principles of perceptual learning and development. New York: Appleton-Century-Crofts, 1969.

Hagen, J. W., \& Hale, G. A. The development of attention in children. In A. D. Pick (Ed.), Minnesota symposia on child psychology (Vol. 7). Minneapolis: University of Minnesota Press. 1973.

Kendler, H. H., \& Kendler, T. S. Vertical and horizontal processes in problem solving. Psychological Review, 1962, 69, 1-16.

Ором, R. D. Effects of perceptual salience on the recall of relevant and incidental dimensional values: A developmental study. Journal of Experimental Psychology, 1972, 92. 285-291.

Odom, R. D., Astor, E. C., \& Cunningham, J. G. Effects of perceptual salience on the matrix task performance of fourand six-year-old children. Child Development, 1975, 46, 758-762.

Oром, R. D., \& Corbin, D. W. Perceptual salience and children's multidimensional problem solving. Child Development, 1973, 44, 425-432.

Odom, R. D., \& Guzman, R. D. Development of hierarchies of dimensional salience. Developmental Psychology, 1972, 6, 271-287.

Piaget, J., \& Inhelder, B. The psychology of the child. New York: Basic Books, 1969.

WEIR, M. W. Developmental changes in problem-solving strategies. Psychological Review, 1964, 71, 473-490.

(Received for publication July 17, 1975.) 